\title{
BMJ Open Village-Integrated Eye Worker trial (VIEW): rationale and design of a cluster- randomised trial to prevent corneal ulcers in resource-limited settings
}

Kieran S O'Brien, ${ }^{1}$ Raghunandan Byanju, ${ }^{2}$ Ram Prasad Kandel, ${ }^{2,3}$ Bimal Poudyal, ${ }^{2}$ Mariya Gautam, ${ }^{2}$ John A Gonzales, ${ }^{1,4}$ Travis C Porco, ${ }^{1,4,5,6}$ John P Whitcher, ${ }^{1,4}$ Muthiah Srinivasan, ${ }^{7}$ Madan Upadhyay, ${ }^{8}$ Thomas M Lietman, ${ }^{1,4,5,6}$ Jeremy D Keenan, ${ }^{1,4}$ The Village-Integrated Eye Worker Trial Group

To cite: O'Brien KS, Byanju R, Kandel RP, et al. VillageIntegrated Eye Worker trial (VIEW): rationale and design of a cluster-randomised trial to prevent corneal ulcers in resource-limited settings. BMJ Open 2018;8:e021556. doi:10.1136/ bmjopen-2018-021556

\section{- Prepublication history for} this paper is available online. To view these files, please visit the journal online (http://dx.doi. org/10.1136/bmjopen-2018021556).

Received 5 January 2018 Revised 25 April 2018 Accepted 30 May 2018
Check for updates

(C) Author(s) (or their employer(s)) 2018. Re-use permitted under CC BY-NC. No commercial re-use. See rights and permissions. Published by BMJ.

For numbered affiliations see end of article.

Correspondence to Dr Jeremy D Keenan; jeremy.keenan@ucsf.edu

\section{ABSTRACT}

Introduction Corneal opacity is a leading cause of blindness worldwide. In resource-limited settings, untreated traumatic corneal abrasions may result in infection and ultimately, opacity. Although antimicrobial treatment of corneal ulcers may successfully cure infections, the scarring that accompanies the resolution of infection can still result in visual impairment. Prevention may be the optimal approach for reducing corneal blindness. Studies have employed community health workers to provide prompt administration of antimicrobials after corneal abrasions to prevent infections, but these studies were not designed to determine the effectiveness of such a programme.

Methods and analysis The Village-Integrated Eye Worker trial (VIEW) is a cluster-randomised trial designed to assess the effectiveness of a community health worker intervention to prevent corneal ulcers. Twentyfour Village Development Committees (VDCs) in Nepal were randomised to receive a corneal ulcer prevention programme or to no intervention. Female Community Health Volunteers (FCHVs) in intervention VDCs are trained to diagnose corneal abrasions, provide antimicrobials and to refer participants when needed. An annual census is conducted over 3 years in all study VDCs to assess the incidence of corneal ulceration via corneal photography (primary outcome). Masked outcome assessors grade corneal photographs to determine the presence or absence of incident corneal opacities. The primary analysis is negative binomial regression to compare the incidence of corneal ulceration by study arm.

Ethics and dissemination The University of California San Francisco Committee on Human Research, Nepal Netra Jyoti Sangh and the Nepal Health Research Council have given ethical approval for the trial. The results of this trial will be presented at local and international meetings and submitted to peer-reviewed journals for publication. Trial registration number NCT01969786; Pre-results.

\section{INTRODUCTION}

Prevention may be the best option for reducing the burden of corneal blindness
Strengths and limitations of this study

- The Village-Integrated Eye Worker Trial is the first randomised controlled trial designed to determine the effectiveness of a community health worker intervention to prevent corneal ulcers.

- The large simple trial design allows detection of a modest intervention effect for a rare outcome.

- Given the nature of the intervention, the study participants and field staff could not be masked.

- The use of corneal photography allows for a masked comparison of the primary outcome.

- Cluster randomisation reduces the risk of contamination, although contamination of randomisation units is still possible in this design. The extent of contamination will be measured with process indicators.

caused by corneal ulcers. Corneal opacity is the fourth leading cause of blindness worldwide, with a disproportionate burden borne by low-income and middle-income countries. ${ }^{1-3}$ In such settings, corneal abrasions that occur as a result of agricultural trauma often go untreated, increasing the chances of a bacterial or fungal corneal ulcer and subsequent opacity. ${ }^{4-8}$ Even successful antimicrobial treatment often leaves a patient with visual impairment because of the associated immune response and resultant corneal scar. ${ }^{9-11}$ Delays in presentation and initiation of antimicrobial therapy result in worse clinical outcomes, including severe visual impairment and corneal perforation. ${ }^{12-15}$ Indeed, the ultimate visual outcome depends less on the specific antimicrobial or anti-inflammatory agent used, and far more on the visual acuity at the time antimicrobial therapy is started. ${ }^{916}$ Reducing the delay in starting antimicrobial therapy 
following a corneal abrasion could prevent infections from developing, and thus could be the best way to reduce corneal opacity-related vision loss in resourcepoor settings.

A promising approach for corneal ulcer prevention is the use of community health workers to diagnose corneal abrasions and provide prompt administration of antimicrobials. Community-level interventions have the potential to increase service uptake in settings with poor access to the healthcare system and may reduce delays in seeking treatment. ${ }^{17}$ Community-based approaches are feasible for eye diseases, with notable successes demonstrated by mass drug administrations for onchocerciasis and trachoma. ${ }^{18-22}$ Several studies have found low rates of infectious keratitis after implementing community health worker programmes for prophylaxis of corneal abrasions. ${ }^{23-26}$ However, these studies were unable to assess the causal impact of the programmes, since all participants were included in the interventions. A cluster-randomised trial would provide the strongest form of evidence for the effectiveness of a community health worker programme for corneal ulcer prevention.

Designing a randomised trial to determine the effectiveness of a corneal ulcer prevention programme is challenging. Corneal ulceration is a relatively rare outcome, with estimates ranging from 11 cases per 100000 personyears in Minnesota to 799 cases per 100000 personyears in Nepal. ${ }^{2324}$ 27-30 A very large sample size would be needed to enrol enough cases to detect an effect. Furthermore, corneal ulcer detection is difficult. Previous studies have relied on programme referrals, but in a trial setting with a control group, this approach is prone to bias. ${ }^{23-26}$ Clinic-based case finding would likely underestimate the true number of corneal ulcers in settings with poor access to healthcare. This approach could also result in bias between the groups, with a paradoxically higher number of patients in the intervention group due to increased attention and referrals. In addition, a successful programme would require publicity and education, which might be difficult to administer in a randomised fashion to a public at risk for a disease but not yet afflicted and may result in contamination.

In the present report, we describe the methods of a cluster-randomised trial that uses a large simple trial design to overcome these challenges. ${ }^{31}$ The Village-Integrated Eye Worker (VIEW) trial is a cluster-randomised trial designed to determine the effectiveness of a community health worker-based intervention to prevent corneal ulcers. Community randomisation protects against the risk of contamination posed by an individual-randomised trial, increases feasibility of intervention delivery and is well suited for the nature of a corneal ulcer prevention intervention. A simple outcome (incidence of corneal ulceration), assessed identically within the intervention and control communities using electronic data capture and smartphone-based photography, allows a large sample size and sufficient statistical power to detect a modest treatment effect for a rare outcome.

\section{METHODS AND ANALYSIS}

\section{Study overview}

In the VIEW trial, Village Development Committees (VDCs) in rural and semi-urban Nepal were randomised in a 1:1 ratio to intervention or control. In communities randomised to the intervention, existing Female Community Health Volunteers (FCHVs) are trained to diagnose corneal abrasions and provide antimicrobial ointments as prophylaxis. An active publicity campaign in intervention communities encourages residents to present to the community health worker within 24 hours of ocular trauma. In control communities, existing FCHVs receive no additional training and no publicity campaign is conducted. No changes to existing eye healthcare services are otherwise made, and residents from both arms are free to seek care at any local healthcare facilities for eye complaints. Masked outcome assessors perform an annual census in both intervention and control communities over a 3-year period. Census workers photograph both corneas of all residents on enrolment into the study and at the fourth annual census, and at any intervening census in which a resident reports symptoms consistent with a corneal ulcer. Corneal photographs are later graded for corneal opacity by masked examiners. An overview of study procedures and study timeline is provided in table 1 .

\section{Specific aims and outcomes}

The specific aims of this trial are (1) to determine whether diagnosis and prophylaxis of corneal abrasions by community health workers will reduce the incidence of corneal ulceration in rural Nepal, (2) to assess the cost-effectiveness of the corneal ulcer prevention programme and (3) to estimate the true incidence of corneal ulceration in this population. We hypothesise that communities in which community health workers are available to provide diagnosis and prophylaxis for corneal abrasions will have a significantly lower incidence of corneal ulceration compared with communities without this service. The primary outcome (specific aim 1) is incident corneal opacity in an individual during the 3-year study period, as determined from corneal photography. 'Incident corneal opacity' is defined as the absence of photographic evidence of a corneal opacity at one census visit followed by the presence of photographic evidence of an opacity at a subsequent visit. Secondary outcomes include (1) the prevalence of visual impairment caused by corneal ulceration as assessed through clinical exams of residents with incident corneal opacities, (2) time from ocular trauma until presentation to the FCHV and (3) awareness of the intervention among the study population as assessed through an annual survey.

\section{Setting and eligibility}

We are conducting this study in all communities from 24 VDCs (government-defined administrative units) in the Chitwan and Nawalparasi districts of Nepal. VDC-level eligibility criteria include location within the catchment 
Table 1 Timeline of major study procedures*

\begin{tabular}{|c|c|c|}
\hline Time point & Activity & Description of activities \\
\hline Month 0 & Phase 0 census (baseline) & $\begin{array}{l}\text { In all } 24 \text { study VDCs, collect the following data: } \\
\text { Demographics } \\
\text { Ocular history screening (lifetime) } \\
\text { Bilateral photography of all participants }\end{array}$ \\
\hline Month 6 & Randomisation & $\begin{array}{l}\text { Randomise } 12 \text { VDCs to receive intervention and } 12 \text { VDCs to receive no } \\
\text { intervention }\end{array}$ \\
\hline Month 8 & Intervention awareness survey & $\begin{array}{l}\text { In all } 24 \text { study VDCs, conduct a survey of a random sample of households to } \\
\text { assess level of awareness of the intervention in both study arms }\end{array}$ \\
\hline Month 12 & Phase 12 census & $\begin{array}{l}\text { In all } 24 \text { study VDCs, collect the following data: } \\
\text { Demographics/vital statistics update } \\
\text { Ocular history screening (past } 12 \text { months) } \\
\text { Symptom-based photography }\end{array}$ \\
\hline Month 20 & Intervention awareness survey & $\begin{array}{l}\text { In all } 24 \text { study VDCs, conduct a survey of a random sample of households to } \\
\text { assess level of awareness of the intervention in both study arms }\end{array}$ \\
\hline Month 24 & Phase 24 census & $\begin{array}{l}\text { In all } 24 \text { study VDCs, collect the following data: } \\
\text { Demographics/vital statistics update } \\
\text { Ocular history screening past } 12 \text { months) } \\
\text { Symptom-based photography }\end{array}$ \\
\hline Month 32 & Intervention awareness survey & $\begin{array}{l}\text { In all } 24 \text { study VDCs, conduct a survey of a random sample of households to } \\
\text { assess level of awareness of the intervention in both study arms }\end{array}$ \\
\hline Month 36 & Phase 36 census (final) & $\begin{array}{l}\text { In all } 24 \text { study VDCs, collect the following data: } \\
\text { Demographics/vital statistics update } \\
\text { Ocular history screening (past } 12 \text { months) } \\
\text { Bilateral photography of all participants }\end{array}$ \\
\hline
\end{tabular}

${ }^{*}$ Census photograph grading and the 12-month visit began after the month 12 census and continued on an ongoing basis.

FCHV, Female Community Health Volunteer; VDC, Village Development Committee.

area of the Bharatpur Eye Hospital and population of $<15000$ per the 2001 government census. Of 112 VDCs in these districts, 24 meet these eligibility criteria and are included in the trial. Geographic separation was not considered in selection of eligible VDCs. All residents in study communities are offered enrolment in each annual census. A census worker visits each household in each village included in the study. At the baseline visit, verbal consent from each head of household was obtained for participation of all household members in the census visits. Data collection for the baseline visit began in January 2014.

\section{Randomisation and masking}

After the baseline census, VDCs were randomised with stratification by district (Chitwan vs Nawalparasi) to receive the intervention or no intervention. Stratification is performed to minimise the chances of bias that could have occurred if the randomisation had been unbalanced between the two geographically distant sets of communities. The study biostatistician generated the random allocation sequence using R (R Foundation for Statistical Computing, Vienna, Austria). Allocation is concealed by enrolling all communities before randomisation and offering the intervention to all community members. Study staff from the Bharatpur Eye Hospital are responsible for implementation of the randomisation sequence.

Due to the nature of the intervention, FCHVs in intervention VDCs are not masked to treatment allocation. The District Chief Public Health Officers, who oversee FCHVs in both intervention and control VDCs, are informed of the study arms. Personnel who perform census activities are unaware of treatment allocation. In addition, all study personnel conducting photograph grading are masked to treatment allocation. These photograph graders are crucial to mask since they are assessing the primary outcome of the trial. The photograph graders are also the easiest to mask, since photographs can be displayed in a random order without identifying information.

\section{Intervention}

Female Community Health Volunteers

The FCHV programme was initiated by the government of Nepal in $1988 .{ }^{32}$ The programme aims to link communities to healthcare and to provide community-based services and health promotion. ${ }^{33}$ FCHVs are selected by their communities, live in the wards they serve and have experience implementing community 
health projects, including family planning and immunisation campaigns. ${ }^{33}{ }^{34}$ Existing FCHVs in intervention VDCs are trained as part of the corneal ulcer prevention programme.

\section{Training and supervision}

FCHVs located in VDCs randomised to the intervention attend a 3-day training course at Bharatpur Eye Hospital. The initial training includes both lecture and hands-on practice. Lecture includes basic eye anatomy, common eye diseases and the difference between ocular trauma, corneal abrasion and corneal ulcer. FCHVs are trained to diagnose corneal abrasions using fluorescein strips, 2.5x magnifying loupes and a light-emitting diode (LED) ultraviolet (UV) flashlight. FCHVs are also trained to measure counting fingers visual acuity, to administer eye ointments, and to enter data into study logbook forms.

Training is conducted in Nepali by trained study staff from the Bharatpur Eye Hospital and is supervised by the investigators. A quiz is administered at the end of the initial 3-day training. FCHVs with scores of $80 \%$ or greater are invited to begin intervention work immediately, whereas those with scores of $<80 \%$ complete additional one-on-one training with the study staff. Illiterate FCHVs are asked to bring a family member or neighbour to the trainings to provide reading and writing support for the data entry portion of the programme.

The study team visits each FCHV weekly to review corneal abrasion cases, collect logbook data and replenish supplies. The study team conducts refresher trainings to review the basic concepts and skills required in diagnosing corneal abrasions. Brief refresher trainings are conducted monthly and more in-depth trainings are conducted every 6 months.

\section{Corneal ulcer prevention programme}

If a participant presents with ocular trauma, redness and/or pain and is interested in participating in the study, written consent is obtained before procedures are performed. If a participant is illiterate, thumbprints are obtained in the presence of a witness. Minors (participants aged $<18$ years of age) and a parent or legal guardian both provide written consent. If the resident does not provide consent, the FCHV may still provide diagnosis and prophylaxis or referral, but she will not record any data onto the logbook form.

Any person presenting to the FCHV with ocular trauma, redness and/or pain is offered a corneal examination, which involves the application of fluorescein to the affected eye (s) and examination with $2.5 \mathrm{x}$ magnifying loupes and an LED UV flashlight to identify the presence of a corneal abrasion. Participants are immediately referred to Bharatpur Eye Hospital or the nearest primary eye care centre if they are diagnosed with a corneal ulcer, bilateral corneal abrasions, visual acuity worse than counting fingers in the unaffected eye or some other ocular abnormality that the FCHV cannot diagnose. Participants with a corneal abrasion receive nine single-dose applicaps of
$1 \%$ chloramphenicol ointment (Chloromycetin Kaps, Pfizer, India) and $1 \%$ itraconazole ointment (Itral, Jawa Pharmaceuticals) to be applied three times daily for 3 days. Pregnant women are given $1 \%$ azithromycin ointment (Zaha, Ajanta Pharma) instead of chloramphenicol. The FCHV applies the first dose to demonstrate the technique, and the remaining eight doses are performed by the participant without direct observation. The FCHV enters information about the participant into, including the participant's demographic information and telephone number, questions about risk factors for ocular trauma, date and time of presentation and of ocular trauma, visual acuity and follow-up visit status. After 3 days, the participant is requested to return to the FCHV for a follow-up examination. At the follow-up visit, participants report the number of doses of medication they used and answer an open-ended question about adverse events. Participants are asked to bring their used applicap containers and ointment tubes to the follow-up visit in order to corroborate reported adherence. The eye is re-examined with fluorescein using the same technique as before. If an allergic reaction, corneal abrasion or corneal ulcer is found on the follow-up examination, the participant is referred to Bharatpur Eye Hospital or the nearest primary eye care centre.

FCHVs will not refuse diagnosis or prophylaxis to anyone based on their residence, even if participants present from control VDCs. FCHVs will record the VDC of all people who present, which will allow us to assess the level of contamination.

\section{Publicity}

Study staff at Bharatpur Eye Hospital hold orientation meetings with teachers, traditional healers and local political leaders to introduce the programme and to encourage community leaders to advertise the programmes. FCHVs in study communities advertise their services for ocular trauma through door-to-door visits with households in their wards and monthly meetings with their ward-level Mother's Groups. FCHVs encourage the community to present to them within 24 hours of experiencing ocular trauma. FCHVs also post advertisements describing ulcer prevention throughout the community and distribute pamphlets, greeting cards and calendars describing the programme. All public publicity materials such as posters are removed prior to the annual census to maintain masking of the census workers. Publicity activities will be limited to the confines of the VDC boundaries in order to prevent contamination.

\section{Outcome assessments}

\section{Census and photography}

Demographics and screening questions. An annual census is conducted in all study communities over the 3-year study period. The baseline census was conducted before randomisation. Census workers visit each household in each study community. After obtaining verbal consent from the head of household, the census worker records 
the full name, age and gender of each household member. The census worker also asks each household member several ocular history questions to determine which household members might have had a corneal ulcer. During interim census periods, the ocular history screening questions refer to experience of ocular symptoms within the past year; the first time the questions are asked, they refer to lifetime experience. The questions include experience of ocular trauma, sudden decreased vision, eye pain and corneal infection. Data are recorded using a custom-designed mobile application on Google Nexus 5 smartphones. Census workers use the mobile device to record the global positioning system (GPS) coordinates of every household to increase efficiency of follow-up activities and allow assessment of spatial relationships.

Corneal photographs. At the baseline and final census phases, the census workers photograph the corneas of all residents in the study area. During the interim census phases, the census workers photograph the corneas of those who answer one or more of the ocular screening questions affirmatively. A smartphone attachment, the Ocular CellScope (Development Impact Lab, Berkeley, California, USA), is used to improve the quality of corneal photographs. ${ }^{35}$ The CellScope is a three-dimensional-printed device with a +25 Diopter lens and external illumination, which allows the smartphone camera to capture high-quality corneal photographs.

Training. Census workers attend a 3-day training at Bharatpur Eye Hospital prior to the start of each annual census. Training includes lectures, hands-on practice with the mobile application and photography and field practice in a non-study community. The study team monitors each census worker weekly to confirm quality and completeness of data collection and photography. Data collection progress is monitored locally by study staff at Bharatpur Eye Hospital and by investigators at the University of California, San Francisco (UCSF) on Salesforce.com.

\section{Photograph grading}

Definitions. Photographs from individuals answering affirmatively to any of the screening questions at a follow-up census are presented for grading in a random order. The grading programme presents all photos for a single eye at a time. Graders are masked to the grades and images of the contralateral eye, to study identifier and to other graders' grades. After indicating whether all photographs were taken of the same eye, the photographs are graded for quality (good, poor but readable or unreadable), and then for the presence of an opacity (definitely yes, probably, possibly, definitely no). For any photograph graded as a possible, probable or definite opacity, the photographs from all preceding phases are presented for comparison. These previous photos are graded according to the same criteria. A random sample of photos stratified by the initial opacity grade is presented a second time to determine intrarater reliability.
Ophthalmologist adjudication. Photographs proceed through several rounds of grading. The process starts with two graders in Nepal independently grading all eligible corneal photographs. Photographs graded as possible, probable or definite opacity by either grader are then presented to one of three cornea specialists for a first round of adjudication. In addition, a random sample of photographs graded as definitely having no opacity or as unreadable quality are sent for the initial adjudication. Any photograph judged to be a possible, probable or definite opacity at this first level of adjudication, as well as any photographs graded as definitely no for opacity by the first adjudicator but probable or definite opacity by both of the initial graders, is subsequently sent to all three ophthalmologists for a second level of adjudication. If two of the three ophthalmologists grade an eye as having a probable or definite opacity at one phase, and definitely no opacity or a possible opacity at a previous phase, the eye will be classified as having an incident opacity for the primary outcome.

Training. Photograph graders and adjudicators receive extensive training. The training includes an overview of the anatomy of the cornea and the pathophysiology of corneal infections. During the training, each photograph grader is presented with 100 cornea photographs taken with the Ocular CellScope, half of which are of corneal ulcers or corneal scars, and half of which are of normal corneas. The results of this exercise are compared against an expert consensus reference grade, which was determined using the consensus grade from three ophthalmologists. Discrepancies are reviewed in person with the photograph graders. Graders who achieve a Cohen's kappa $>0.7$ for inter-rater reliability (comparing the 100 grades against the expert grader) are certified as graders. Graders are retrained and recertified each year, using a different set of corneal photographs.

\section{Twelve-month nested case-control study}

Design. A nested case-control study is conducted among incident cases of corneal ulcer and an equal number of age-matched ( \pm 2 years), sex-matched and community-matched controls, with the visit scheduled to take place 12 months after the case's symptoms started. Visits are preferably conducted at Bharatpur Eye Hospital, Kawasoti Eye Care Center or Parsa Eye Care Center; if participants cannot attend one of these facilities then a mobile team will visit the participant at their home. An eye examination is performed for each eye by an optometrist or ophthalmic assistant, and risk factors for corneal ulceration and quality of life are assessed with standardised questionnaires. Study personnel conducting the $12 \square$ month visit are masked to case-control status of the participant as well as randomisation arm during the procedures.

Clinical examination. Trained study personnel perform manifest refraction and best spectacle-corrected visual acuity assessments, followed by an eye examination and corneal photography. The eye examination is performed 
with a slit lamp biomicroscope in the eye clinics, and with a penlight at the mobile examinations. Based on the eye examination, the examiner states the condition accounting for visual acuity worse than 20/20 (eg, corneal opacity, cataract, glaucoma, etc). In addition, the examiner compares the vision in the worse-seeing eye with the better-seeing eye, and determines the ocular condition responsible for the decrement in the worse-seeing eye (eg, corneal opacity, cataract, glaucoma, etc).

Instruments. The Euroqol-5D $\square 5 \mathrm{~L}$ Quality of Life Questionnaire and Hong Kong visual functioning questionnaires were translated from English to Nepali and back-translated independently by two bilingual study staff members at the Bharatpur Eye Hospital. A committee reviewed the questionnaires to determine the appropriateness of the questions for this population and pilottested the refined questionnaires on a sample of patients at the Bharatpur Eye Hospital. Questionnaires on risk factors for corneal ulcers include questions on agricultural trauma, contact lens wear and use of topical corticosteroids, as well as healthcare seeking behaviours after eye trauma. The costing questionnaire elicits all patient-related and hospital-related costs of the corneal opacity, including laboratory testing, medications and surgeries, as well as the opportunity costs of attending hospital visits.

\section{Clinic-based case finding}

Clinic $\square$ based case finding is conducted at several sites throughout the study area. These sites were identified as locations that receive corneal abrasion and corneal ulcer cases. Each month, study staff visit each of these sites and use the site's patient logbook to record data on any corneal abrasion, corneal ulcer or corneal foreign body case that presented to that site in the past month.

\section{Intervention awareness surveys}

An intervention awareness survey is conducted annually in all VDCs, with survey workers not informed about the trial intervention, and masked to whether the community has been randomised to intervention or control. A random sample of households from the most recent census is selected to participate in the survey. Census data, including name, phone number and household GPS coordinates, are uploaded to the mobile software platform GIS Cloud (GIS Cloud, London, UK, http:// www.giscloud.com) for the survey. The trained survey workers use handheld mobile devices to identify households from a map generated by the application, and ask an adult in the household a series of questions designed to determine their level of awareness of the intervention. Survey workers are required to complete the survey on at least 10 of the 15 selected households in each ward.

Conducting the intervention awareness surveys in control VDCs will provide a measure of contamination. Publicity is limited to intervention VDCs to reduce the likelihood of contamination, but it is possible that residents of control VDCs will learn of the intervention through exposure to publicity materials or word of mouth. Any awareness of the intervention found in control VDCs will be indicative of contamination.

An unmasked intervention awareness survey is conducted by trained study personnel annually in intervention VDCs only. Unlike the masked survey, survey workers understand the nature of the intervention and publicity campaign, and provide additional information about the programme if the resident is unaware of the intervention. The unmasked survey is conducted identically to the masked survey in terms of selection of households and data collection and management, but provides information about the impact of the intervention sooner, so that corrective actions can be taken.

\section{Programmatic costs}

All programmatic costs, including staff salaries, equipment, antimicrobial ointments, outreach and advertising and training costs are recorded by the study coordinator during the trial. Costs are collected by the study coordinator for each year of the programme.

\section{Data collection, management, monitoring}

All electronic data and photographs are uploaded daily to secure, cloud-based servers. Data collected on paper are double-data entered and adjudicated in REDCap. ${ }^{36}$ Study personnel collecting data receive at least a 1 day initial training, and periodic refresher trainings. Study staff at Bharatpur Eye Hospital conduct weekly monitoring visits to all FCHVs, census workers and survey workers collecting data, and data collection progress is reviewed by the data manager at UCSF weekly.

\section{Data and Safety Monitoring Committee}

The Data and Safety Monitoring Committee (DSMC) for this trial includes independent experts in bioethics, biostatistics, epidemiology, ophthalmology and international public health appointed by the National Eye Institute-National Institutes of Health and empaneled before the start of the study. The DSMC meets at least once each year, and organises teleconferences as needed for progress reporting. The study protocol and modifications are subject to review and approval by Institutional Review Boards at UCSF and in Nepal, and by the DSMC. The DSMC monitors severe or unexpected events that threaten the safety of patients and oversees the data collected throughout the duration of the study. The DSMC is responsible for reviewing the results of the interim analysis and determining whether or not the trial should continue, with or without modifications.

\section{Statistical analysis plan}

Sample size

Sample size and power calculations are based on an estimated incidence of corneal ulceration of 100 per 100000 person-years. We estimate that 12 VDCs per arm will provide $>80 \%$ power to detect a $30 \%$ reduction in incidence of corneal ulceration, assuming 9000 people per VDC, an intraclass correlation coefficient of 0.00015 and a two-tailed alpha of 0.05 . 
Table 2 Trial registration data and protocol summary

\begin{tabular}{|c|c|}
\hline Data category & Information \\
\hline Primary registry and trial identifying number & ClinicalTrials.gov NCT01969786 \\
\hline Date of registration in primary registry & 25 October 2013 \\
\hline Source(s) of monetary or material support & National Eye Institute-National Institutes of Health \\
\hline Primary sponsor & National Eye Institute-National Institutes of Health \\
\hline Contact for queries & Thomas M Lietman, MD (tom.lietman@ucsf.edu) \\
\hline Title & Village Integrated Eye Worker trial \\
\hline Countries of recruitment & Nepal \\
\hline Health condition(s) or problem(s) studied & Corneal ulcer prevention \\
\hline Intervention(s) & $\begin{array}{l}\text { Intervention: training volunteer community health workers to diagnose corneal } \\
\text { abrasions and provide antimicrobial ointment to prevent corneal ulcers } \\
\text { Control: no intervention }\end{array}$ \\
\hline Study type & Cluster-randomised trial \\
\hline Date of first enrolment & January 2014 \\
\hline Target sample size & 24 Village Development Committees, 216000 individuals \\
\hline Recruitment status & Recruiting \\
\hline Primary outcome(s) & Incidence of corneal ulcer (time frame: 3 years) \\
\hline Key secondary outcomes & Trial-based cost-effectiveness analysis \\
\hline
\end{tabular}

Interim analysis and stopping guidelines

An interim analysis for efficacy is performed one-third of the way through the trial, with alpha set at 0.001 . The interim analysis has approximately $70 \%$ power to detect a $68 \%$ reduction in corneal ulcer rates over the single year. The DSMC reviews the unmasked interim analysis and makes recommendations on the continuation of the trial. No interim analysis for futility is performed.

\section{Specific aim 1}

The primary analysis is negative binomial regression to compare the incidence of corneal ulcers between treatment arms, with the count of incident corneal ulcers over the study period as the outcome, log person-time at risk as an offset and treatment arm as the sole covariate. An individual is determined to have an ulcer if a new opacity is identified by photograph grading at a follow-up census. For individual-level data, an opacity identified at a follow-up census will be considered new if it is absent on a photograph of acceptable quality from at least one previous census. For community-level data, we will compute the total count of new opacities identified in each randomisation unit at each of the follow-up census phase. Individuals can contribute multiple incident ulcers to the overall count, but no more than one new opacity per eye per phase. Individuals start contributing person-time at the first census they are photographed and continue contributing person-time until the final census with complete data (ie, screening questions answered, and if required, then photographs taken and uploaded). Individuals who develop ulcers will continue to contribute person time and can contribute additional ulcers until they leave the study (ie, permanently moved, died or study conclusion).

Negative binomial regression explicitly addresses the cluster-randomised nature of the design, and the proposed analysis follows the intent-to-treat principle. We will use a permutation $\mathrm{p}$ value, taking into account the stratified design of the randomisation.

\section{Specific aim 2}

The primary analysis is a trial-based cost-effectiveness analysis of the costs per corneal ulcer prevented, assessed at the VDC level. Costs include all programmatic and treatment costs per VDC over the duration of the 3-year study. The effectiveness outcome will be the same as for specific aim 1: the number of incident corneal opacities per VDC. Both costs and effects will be discounted at $5 \%$ per year for the 3-year time horizon of the analysis. We will use the non-parametric bootstrap to estimate the joint sampling distribution of the differences in average VDC costs and effects between the treatment arms, and plot this on the cost-effectiveness plane and in a cost-effectiveness 
acceptability curve. In a secondary analysis, we convert visual acuity data from the 12-month visit into quality-adjusted life-years (QALYs), and perform the same analyses in terms of costs per QALYs lost. We will also conduct a hypothetical cohort-based cost-effectiveness analysis as a supplement to assist in interpretive generalisation beyond the specific programmatic cost structure of the Nepal trial setting.

\section{Specific aim 3}

The primary analysis is an assessment of the true incidence of corneal ulceration in the control arm. The primary outcome of the trial (specific aim 1) will produce an estimate of the incidence of corneal ulceration sufficient to answer the overall research question, but the estimate itself may be biased by outcome misclassification and missing data. The use of census photographs alone as the outcome will result in some number of false negatives and false positives. Despite rigorous efforts to ensure high coverage during census phases, it is not possible to capture every single person, thus it is possible to miss incident ulcers using only census photographs as the outcome. In addition, the grading process inevitably results in some photos falsely classified as having an opacity. Internal validation data collected during the 12-month nested case-control study will be used to correct the estimate of the incidence in the control arm for misclassification.

We will report community-stratified, age-stratified and gender-stratified incidence rates. The spatial distribution of incident corneal ulcers will be examined using coordinates obtained at the time of the census. The association between incident corneal ulceration and individual-level risk factors collected at the time of the census, including age, sex and urban/rural residence, will be assessed with clustered logistic regression.

\section{Patient and public involvement}

Focus groups were conducted among residents of randomly selected communities within the study area to identify local eye health resource utilisation patterns and understand the language used to describe ocular trauma. Summaries of focus group discussions are used to inform training programmes for census workers, intervention awareness survey workers and publicity campaigns. Feedback from FCHVs participating in the training programme is used to improve refresher trainings.

\section{Ethics and dissemination}

The District Public Health Offices of the Nawalparasi and Chitwan districts provide approval for the study each year before census data collection commences. The trial is registered at clinicaltrials.gov (NCT01969786). Protocol modifications are submitted to the relevant parties for review and/or approval. Table 2 summarises the study protocol and trial registration information.

Verbal consent is obtained for census and photography, awareness surveys and the 12-month follow-up visit. Written consent is obtained for FCHV-administered medications. Data and photos collected on individuals are linked to individual participant information using unique identifiers. Only key study personnel have access to identifying information.

The results of this trial will be presented at local and international meetings and submitted to peer-reviewed journals for publication.

\section{Author affiliations}

${ }^{1}$ Francis I. Proctor Foundation, University of California, San Francisco, San Francisco, California, USA

${ }^{2}$ Bharatpur Eye Hospital, Bharatpur, Nepal

${ }^{3}$ Seva Foundation, Berkeley, California, USA

${ }^{4}$ Department of Ophthalmology, University of California, San Francisco, California, USA

${ }^{5}$ Department of Epidemiology and Biostatistics, University of California San Francisco, San Francisco, California, USA

${ }^{6}$ Institute for Global Health Sciences, University of California San Francisco, San Francisco, California, USA

${ }^{7}$ Aravind Eye Care System, Madurai, India

${ }^{8}$ BP Eye Foundation, Children's Hospital for Eye, Ear, and Rehabilitation Services (CHEERS), Kathmandu, Nepal

Acknowledgements The authors would like to thank the District Public Health Offices (Chitwan and Nawalparasi, Nepal) and Nepal Health Research Council (Kathmandu, Nepal) for helping with study coordination and implementation. The authors would like to thank the partners at the Seva Foundation, especially Suzanne Gilbert, Ken Bassett, Chundak Tenzing, Heidi Chase, Parami Dhakhwa and Shravan Chaudhary. The authors are grateful for the guidance provided by the DSMC members, including William Barlow (chair), Patricia Buffler, Kavita Dhakhwa, Leslie Hyman, Art Reingold, Serge Resnikoff, Larry Schwab and Carrie Thiessen, as well as our NIH Program officer Don Everett. The authors would also like to thank Sun Y Cotter for her integral role in study implementation.

Collaborators M. Srinivasan (Aravind Eye Care System, Madurai, India); Manmohan Adhikari, Gopal Bhandari, Sadhan Bhandari (Assistant Study Coordinator), Dipak Bhattarai (Supervisor), Rabin Bhattarai (Supervisor), Dikshya Bista, Raghunandan Byanju (Investigator), Dipak Chapagain (Supervisor), Ajay Kumar Chaudhary (Supervisor), Daya Shankar Chaudhary, Gokul Dahal, Mariya Gautam, Shree Krishna Gautam (Supervisor), Ramesh Ghimire, Gaurav Giri, Puspa Giri, Dhanmaya Gurau (Supervisor), Jeevan Gurung (Assistant Study Coordinator), Vivek Hamal, Deepak Kandel (Supervisor), Sajani Kayastha, Kamal Bahadur Khadka (Investigator), Pradip Chandara Lamichhane (Supervisor), Tulsi Prasad Parajuli, Bimal Poudyal (Study Coordinator), Ranjeet Kumar Shah, Niraj Sharma, Prafulla Sharma, Anju Shrestha, Manisha Shrestha, Pradeep Subedi, Rajendra Rijal (Supervisor) (Bharatpur Eye Hospital, Bharatpur, Nepal); Madan Upadhyay (BP Eye Foundation/Children's Hospital for Eye, Ear, and Rehabilitation Services, Kathmandu, Nepal); Bill Barlow, Patricia Buffler, Kavita Dhakhwa, Leslie Hyman, Art Reingold, Serge Resnikoff, Larry Schwab , Carrie Thiessen (Data and Safety Monitoring Committee); Nisha Acharya, Sun Cotter, John Gonzales, Jeremy Keenan (Investigator), Jessica Kim, Salena Lee, Thomas Lietman (Principal Investigator), Stephen McLeod, Kieran 0'Brien (Study Coordinator), Travis Porco (Biostatistician), David Ramirez, Kathryn Ray, Jennifer Rose-Nussbaumer, Jack Whitcher (Francis I. Proctor Foundation, University of California, San Francisco, CA, USA); Donald Everett (Program Officer) (National Eye Institute, Bethesda, MD, USA); Krishna Raj Dharel, Tejendra Bdr Khadka, Chij Kumar Maskey, Sailesh Kumar Mishra, Tirtha Prasad Mishra, Jaya Ram Shrestha, Laximi Charan Shrestha, Unnat Shrestha (Nepal Netra Jyoti Sangh, Bharatpur and Kathmandu, Nepal); Ken Basset, Heidi Chase, Shravan Choudhary, Parami Dhakwa, Lauren Evans, Suzanne Gilbert, Ram Prasad Kandel, Deborah Moses, Chundak Tenzing (Seva Foundation, Berkeley, CA, USA; Kathmandu, Nepal; Vancouver, BC, Canada).

Contributors KSO'B, TCP, JPW, MS, MU, TML and JDK developed the study design, drafted the protocol and contributed to study implementation and monitoring. $\mathrm{RB}$, $\mathrm{RPK}, \mathrm{BP}, \mathrm{MG}$ and JAG contributed to study implementation and monitoring. The Village Integrated Eye Worker Trial Group contributed to all aspects of this project, including study design, protocol development, regulatory and ethical approval and study implementation and monitoring.

Funding This work was supported by the National Eye Institute of the National Institutes of Health grant number (U10EY022880), the Peierls Foundation and the 
Alta California Eye Research Foundation. The study sponsors had no role in study design; collection, management, analysis or interpretation of data; writing of the report or the decision to submit the report for publication.

Competing interests None declared.

Patient consent Not required.

Ethics approval The study received Institutional Review Board approval from the University of California, San Francisco Committee on Human Research, Nepal Netra Jyoti Sangh and the Nepal Health Research Council.

Provenance and peer review Not commissioned; externally peer reviewed.

Open access This is an open access article distributed in accordance with the Creative Commons Attribution Non Commercial (CC BY-NC 4.0) license, which permits others to distribute, remix, adapt, build upon this work non-commercially, and license their derivative works on different terms, provided the original work is properly cited, appropriate credit is given, any changes made indicated, and the use is non-commercial. See: http:// creativecommons.org/licenses/by-nc/4.0/.

\section{REFERENCES}

1. Pascolini D, Mariotti SP. Global estimates of visual impairment: 2010. Br J Ophthalmol 2012;96:614-8.

2. Whitcher JP, Srinivasan M. Corneal ulceration in the developing world-a silent epidemic. Br J Ophthalmol 1997;81:622-3.

3. Whitcher JP, Srinivasan M, Upadhyay MP. Corneal blindness: a global perspective. Bull World Health Organ 2001;79:214-21.

4. Upadhyay MP, Karmacharya PC, Koirala S, et al. Epidemiologic characteristics, predisposing factors, and etiologic diagnosis of corneal ulceration in Nepal. Am J Ophthalmol 1991;111:92-9.

5. Srinivasan M, Gonzales CA, George C, et al. Epidemiology and aetiological diagnosis of corneal ulceration in Madurai, south India. $\mathrm{Br} J$ Ophthalmol 1997;81:965-71.

6. Gopinathan U, Garg P, Fernandes M, et al. The epidemiological features and laboratory results of fungal keratitis: a 10-year review at a referral eye care center in South India. Cornea 2002;21:555-9.

7. Upadhyay MP, Srinivasan M, Whitcher JP. Microbial keratitis in the developing world: does prevention work? Int Ophthalmol Clin 2007;47:17-25.

8. Srinivasan M, Mascarenhas J, Rajaraman R, et al. The steroids for corneal ulcers trial: study design and baseline characteristics. Arch Ophthalmol 2012;130:151-7.

9. Green MD, Apel AJ, Naduvilath T, et al. Clinical outcomes of keratitis. Clin Exp Ophthalmol 2007;35:421-6.

10. Prajna NV, Mascarenhas J, Krishnan T, et al. Comparison of natamycin and voriconazole for the treatment of fungal keratitis. Arch Ophthalmol 2010;128:672-8.

11. Srinivasan M, Mascarenhas J, Rajaraman R, et al. Corticosteroids for bacterial keratitis: the Steroids for Corneal Ulcers Trial (SCUT). Arch Ophthalmol 2012;130:143-50.

12. Miedziak Al, Miller MR, Rapuano CJ, et al. Risk factors in microbial keratitis leading to penetrating keratoplasty. Ophthalmology 1999;106:1166-71.

13. Kunimoto DY, Sharma S, Garg P, et al. Corneal ulceration in the elderly in Hyderabad, south India. Br J Ophthalmol 2000;84:54-9.

14. Claerhout I, Goegebuer A, Van Den Broecke C, et al. Delay in diagnosis and outcome of Acanthamoeba keratitis. Graefes Arch Clin Exp Ophthalmol 2004;242:648-53.
15. Keay L, Edwards K, Naduvilath T, et al. Factors affecting the morbidity of contact lens-related microbial keratitis: a population study. Invest Ophthalmol Vis Sci 2006;47:4302-8.

16. Lalitha P, Prajna NV, Kabra A, et al. Risk factors for treatment outcome in fungal keratitis. Ophthalmology 2006;113:526-30.

17. Mahande M, Tharaney M, Kirumbi E, et al. Uptake of trichiasis surgical services in Tanzania through two village-based approaches. Br J Ophthalmol 2007;91:139-42.

18. Galega FP, Heymann DL, Nasah BT. Gonococcal ophthalmia neonatorum: the case for prophylaxis in tropical Africa. Bull World Health Organ 1984;62:95-8.

19. Hammerschlag MR, Chandler JW, Alexander ER, et al. Erythromycin ointment for ocular prophylaxis of neonatal chlamydial infection. JAMA 1980;244:2291-3.

20. Mayo-Wilson E, Imdad A, Herzer K, et al. Vitamin A supplements for preventing mortality, illness, and blindness in children aged under 5: systematic review and meta-analysis. BMJ 2011;343:d5094.

21. Taylor HR, Pacqué M, Muñoz B, et al. Impact of mass treatment of onchocerciasis with ivermectin on the transmission of infection. Science 1990;250:116-8.

22. West SK. Blinding trachoma: prevention with the safe strategy. Am J Trop Med Hyg 2003;69:18-23.

23. Upadhyay MP, Karmacharya PC, Koirala S, et al. The Bhaktapur eye study: ocular trauma and antibiotic prophylaxis for the prevention of corneal ulceration in Nepal. Br J Ophthalmol 2001;85:388-92.

24. Getshen K, Srinivasan M, Upadhyay MP, et al. Corneal ulceration in South East Asia. I: a model for the prevention of bacterial ulcers at the village level in rural Bhutan. Br J Ophthalmol 2006;90:276-8.

25. Maung N, Thant CC, Srinivasan M, et al. Corneal ulceration in South East Asia. II: a strategy for the prevention of fungal keratitis at the village level in Burma. Br J Ophthalmol 2006;90:968-70.

26. Srinivasan M, Upadhyay MP, Priyadarsini B, et al. Corneal ulceration in south-east Asia III: prevention of fungal keratitis at the village level in south India using topical antibiotics. $\mathrm{Br} J$ Ophthalmol 2006;90:1472-5.

27. Erie JC, Nevitt MP, Hodge DO, et al. Incidence of ulcerative keratitis in a defined population from 1950 through 1988. Arch Ophthalmol 1993;111:1665-71.

28. Gonzales CA, Srinivasan M, Whitcher JP, et al. Incidence of corneal ulceration in Madurai district, South India. Ophthalmic Epidemiol 1996;3:159-66.

29. Jeng $\mathrm{BH}$, Gritz DC, Kumar $\mathrm{AB}$, et al. Epidemiology of ulcerative keratitis in Northern California. Arch Ophthalmol 2010;128:1022-8.

30. Courtright P, Lewallen S, Kanjaloti S, et al. Traditional eye medicine use among patients with corneal disease in rural Malawi. $\mathrm{Br} J$ Ophthalmol 1994;78:810-2.

31. Yusuf S, Collins R, Peto R. Why do we need some large. simple randomized trials? Stat Med 1984;3:409-22.

32. Global evidence of community health workers for delivery of health related millenium development goals. Geneva, Switzerland: World Health Organization, 2010.

33. Annual report. ed. Services DoH. Government of Nepal: Kathmandu, Nepal: Ministry of Health and Population, 2010.

34. Schwarz D, Sharma R, Bashyal C, et al. Strengthening Nepal's Female Community Health Volunteer network: a qualitative study of experiences at two years. BMC Health Serv Res 2014;14:473.

35. Maamari RN, Ausayakhun S, Margolis TP, et al. Novel telemedicine device for diagnosis of corneal abrasions and ulcers in resource-poor settings. JAMA Ophthalmol 2014;132:894-5.

36. Harris PA, Taylor R, Thielke R, et al. Research electronic data capture (REDCap)--a metadata-driven methodology and workflow process for providing translational research informatics support. J Biomed Inform 2009;42:377-81. 